Laure Gossec Grant/research support from: AbbVie, BMS, Celgene, Janssen, Lilly, MSD, Novartis-Sandoz, Pfizer, Sanofi, and UCB, Consultant for: AbbVie, Biogen, BMS, Celgene, Janssen, Lilly, MSD, Nordic Pharma, Novartis-Sandoz, Pfizer, Roche, Sanofi, and UCB, Consultant for: L Gos$\mathrm{sec}$ has received honoraria from Celgene as investigator for this study, Francis Berenbaum: None declared

DOI: 10.1136/annrheumdis-2019-eular.1592

\section{THU0643 ARE PSORIATIC ARTHRITIS OUTCOMES BETTER IN EARLY ARTHRITIS SERVICE? STUDY FROM A NATIONAL AWARD WINNING CENTRE}

Muhammad Khurram Nisar. Luton and Dunstable University Hospital, Rheumatology, Luton, United Kingdom

Background: There is good evidence that dedicated early arthritis clinics (EACs) improve referral lag time and reduce delay in establishing disease-modifying therapy. However it remains arguable whether such clinics improve outcomes especially for arthritides other than RA. In the UK, only $57 \%$ of units have dedicated EACs. Our early arthritis service won national best practice commendation award for achieving high standards. Objectives: We analysed our psoriatic arthritis (PsA) population data to ascertain whether this cohort benefits from EACs.

Methods: The department set up an early arthritis service with introduction of six clinics (EACs) every week. An agreed treatment protocol incorporating ultrasound was developed to ensure standardised approach to early initiation of treatment, drug education and timely review. This is a retrospective study of all patients with PsA presenting to the service in the first year

Results: Our catchment area covers a population of 350,000 with $40 \%$ ethnic minorities. Of 1884 patients referred, $482(25.5 \%)$ were triaged into EACs based on set criteria. All were reviewed within 3 weeks. 247 $(51 \%)$ were confirmed to have early inflammatory arthritis (EIA). Mean age was 52.4 years (17-86y). 157 (63.5\%) were women. 177 (71.6\%) were White, $58(23.5 \%)$ of Asian and twelve of other background. 159 $(64.3 \%)$ had RA, 55 (22\%) with PsA and 33 had other inflammatory arthritides. There was median 26 weeks delay (0.4-1043 weeks) from symptom onset to GP presentation. Median time for GP referral to the department was 4.0 days (0-84 days).

All PsA patients had regular PsARC assessment. Mean tender (TJ) and swollen joint (SJ) counts at first visit were 8.2 (1-35) and 3.5 (0-14) respectively [n=55]. The patient (PtGA) and physician (PhGA) global assessments mean were 3.0 and 2.9 (1-5)

$95 \%$ commenced their DMARDs within 3 week of initial review. Other $5 \%$ who missed the target was owing to patient factors. Target [TJ \& SJ 52] was achieved for 38 patients $(69 \%)$ and good PsARC response for a further four $(7 \%)$. Median time to achieve the target or good response was 22 weeks (0-48 weeks). Of 55 , only four $(7 \%)$ patients required escalation to biologic therapy. Final TJ and SJ mean was significantly better at $1.2(0-4)$ and $0.3(0-2)[p<.0001]$ with similar improvement in PtGA [mean $1.8(1-4)$ ] and PhGA [mean $1.6(1-3)$ ]. Only six (11\%) patients were true non-responders as the remaining seven declined therapy.

Conclusion: Dedicated EACs help achieve good clinical outcomes in majority of PsA patients. Nearly $76 \%$ of our cohort attained the target or good PSARC response in less than six months. This was despite a significant delay in patients presenting to their GPs and moderately-high disease activity. $100 \%$ of our patients were treated to target facilitated by protocol driven escalation of therapy in these clinics. This is in contrast to the national audit findings whereby only $68 \%$ of patients were treated with disease modifying drugs within 6 weeks of referral and $89 \%$ had treatment to target.

This study shows that the establishment of dedicated EACs improve the prognosis of psoriatic arthritis in terms of primary clinical outcomes compared to patients managed outside of EACs.

Disclosure of Interests: Muhammad Khurram Nisar Grant/research support from: Muhammad Nisar undertakes clinical trials and received support (including attendance at conferences, speaker fees and honoraria) from Roche, Chugai, MSD, Abbvie, Pfizer, BMS, Novartis, Celgene, Mallinckrodt, UCB and Lilly, Consultant for: Muhammad Nisar undertakes clinical trials and received support (including attendance at conferences, speaker fees and honoraria) from Roche, Chugai, MSD, Abbvie, Pfizer, BMS, Novartis, Celgene, Mallinckrodt, UCB and Lilly, Speakers bureau: Muhammad Nisar undertakes clinical trials and received support (including attendance at conferences, speaker fees and honoraria) from Roche, Chugai, MSD, Abbvie, Pfizer, BMS, Novartis, Celgene, Mallinckrodt, UCB and Lilly
DOI: 10.1136/annrheumdis-2019-eular.203

\section{THU0644 PATIENTS' PERCEPTION AND USE OF MEDICAL MARIJUANA}

Kelly Gavigan ${ }^{1}$, Shilpa Venkatachalam ${ }^{1}$, Jeffrey Curtis ${ }^{2}$, Seth Ginsberg ${ }^{1}$, W. Benjamin Nowell'. ' 'Global Healthy Living Foundation, Nyack, United States of America; ${ }^{2}$ University of Alabama at Birmingham, Birmingham, United States of America

Background: Though the introduction of biologics has resulted in significant improvements in their quality of life, people living with rheumatic and musculoskeletal disease (RMD) often seek alternative treatments, such as marijuana (THC) and cannabidiol (CBD). As these substances become more widely available, and legal in some jurisdictions, health care providers (HCP) need to understand patients' THC/CBD perceptions, use and information needs.

Objectives: To examine patient behavior and information needs regarding THC/CBD medical use.

Methods: A 77-item survey was developed in partnership with RMD patient partners and administered online via CreakyJoints and the ArthritisPower research registry. Participants (pts) were eligible if they were $\geq 19$ years of age, resided in the US and reported physician-diagnosed RMD. Pts reported current health status (NIH PROMIS Global Health), use and perceptions of THC/CBD, and related information needs.

Results: To date, 189 pts completed the survey. A majority of pts were female $(87 \%)$ and white $(93 \%)$, with mean age of $55(11)$. More than hal of all pts $(62 \%)$ reported a diagnosis of rheumatoid arthritis. Most pts (78\%) reported fair/poor health (PROMIS Global Physical Health <43). Only $30 \%$ of all pts were satisfied with their current treatment, and more than half $(63 \%)$ had been on their current treatment for $>1$ year. O those surveyed, a majority of pts $(n=168,89 \%)$ reported trying THC and/ or CBD for a purpose they perceived as medical and offered various reasons for initiating its use (Table). Half of all pts $(n=98,52 \%)$ reported ever using CBD and a third $(n=70,37 \%)$ ever using THC "for medical reasons," fifty-one (73\%) of whom currently use THC. More than half (53\%) of those currently using THC reported using it at least once daily. Top reasons for stopping among the 19 who previously used THC were cost $(26 \%)$ and illegality (26\%). Most pts who had ever used THC reported that THC improved their symptoms $(83 \%)$ and/or their condition (71\%). Pain (100\%) and sleep disturbance $(73 \%)$ were the main symptoms pts sought to relieve with THC. Many pts had used THC in lieu of prescribed $(56 \%)$ or OTC $(73 \%)$ medications. Two thirds $(67 \%)$ reported telling their HCP about their THC use, most of whom (64\%) reported that their HCP did not consider it when making treatment changes nor offer advice about mode of administration or dosage. When acquiring THC, $39 \%$ of pts used a medical marijuana card issued by the state the main reason given for not using a card was that THC was not legal for medical use where the pt lived (44\%). Whether they had used THC for medical reasons or not, nearly all pts wanted information about THC, including its effectiveness $(37 \%)$ and its interaction with other medications (34\%); a majority preferred to receive information from HCPs $(55 \%)$ or online educational resources $(34 \%)$. Two thirds $(60 \%)$ of all pts expressed interest in THC/CBD trial participation.

Table: Participants' Reasons for Initiating THC/CBD Use ( $\mathrm{N}=168)$

\begin{tabular}{ll}
\hline Reasons (not mutually exclusive) & $\mathbf{n ~ ( \% )}$ \\
\hline To address symptoms (e.g. pain) experienced despite taking medication & $74(44)$ \\
To address symptoms with less or no medication & $50(30)$ \\
Nothing else worked to treat condition or symptoms & $33(20)$ \\
Friend suggested it & $30(18)$ \\
Saw information online & $28(17)$ \\
Had used marijuana recreationally and wanted to try it for medical reasons & $25(15)$ \\
Medical marijuana became legal in state & $24(14)$ \\
To address side effect(s) from medication & $19(11)$ \\
Physician suggested it & $17(10)$ \\
Relative suggested it & $17(10)$ \\
\hline
\end{tabular}

Conclusion: Though many pts have used or currently use THC/CBD to substitute for or augment their prescribed treatment, pts lack adequate information to guide its use for medical reasons.

Disclosure of Interests: None declared

DOI: 10.1136/annrheumdis-2019-eular.8030 


\section{THU0645 VIRTUAL VISITS IS THE FUTURE COMING? TELEREUMATOLOGY. PILOT PROJECT: REVIR PROGRAM, RHEUMATOLOGY SERVICE. BARCELONA, SPAIN}

Fabiola Ojeda ${ }^{1,1}$, Manel Ciria ${ }^{1}$, Carolina Perez-Garcia ${ }^{1}$, Eric Sitjas ${ }^{2}$

Elena Martinez ${ }^{3}$, Daniel Martinez-Laguna ${ }^{4}$, Montserrat Pimienta ${ }^{5}$, Jordi Monfort ${ }^{1}$. ${ }^{1}$ Hospital del Mar, Rheumatology, Barcelona, Spain; ${ }^{2}$ Hospital del Mar, Direction, Barcelona, Spain; ${ }^{3}$ Institut Català de la Salut, CAP Vila Olímpica, Barcelona, Spain; ${ }^{4}$ Institut Català de la Salut, CAP Sant Martí, Barcelona, Spain; ${ }^{5}$ Institut Català de la Salut, CAP Ramón Turró, Barcelona, Spain

Background: The prevalence of rheumatic diseases in the Spanish population is estimated at $22.6 \%$ according to the EPISER 2000 study. A new epidemiological study is currently underway, EPISER 2016 and its preliminary results point towards a higher prevalence. Within the national health system, primary care is the first level of access to the health system and is provided at primary care centers (CAP). The rheumatology at primary care centers at Spain has been a pioneer in the application from the team at Parc de Salut Mar (Hospital del Mar), with the physical presence of rheumatologists in the 14 centers belonging to the CAP network of the SAP Litoral. The high prevalence of the medical pathology of the musculoskeletal syste and the aging of the population, can condition an increase in visit requests in rheumatology, and with it, the increase in the waiting lists of patients.

Objectives: The main objective of this study is to know the resolutive possibility of virtualization, measured in the number of visits resolved telematically, as well as its impact on the resolution capacity of the primary care physician and the reduction of the waiting list of first face-to-face visits.

Methods: Prospective experimental study, started on December 1, 2017 and ended on May 31, 2018. Four primary care centers were selected according to population and waiting list: Sant Martí Nord, Sant Martí Sud, Ramón Turró and Villa Olímpica. The REVIR program proposes the creation of a circuit for the assessment of referrals to rheumatology from primary care physicians (MAP).

Results: 726 first visit requests were received during the REVIR program. The most common categorized pathology was mechanical pathology, representing about $70 \%$ of the first visits requested. Metabolic bone disease ranked second with $16 \%$, and inflammatory pathology ranked third (SLE, RA, SA, SPA, PsA). Chronic musculoskeletal pain ranked fourth (including fibromyalgia) and lastly soft tissue pathology. The number of first visit requests was multiplied by two in all the participating primary care centers of the project. Despite this increase, the telematic resolution of the visits created was stable, with a value greater than $40 \%$.

Conclusion: The implementation of a system of assessment of the first visits in rheumatology requested from Primary Care is effective in decreasing the waiting list to make face-to-face visits, as well as to detect early serious pathology that requires hospital control. It has been achieved, therefore, that the patient is treated at the level of attention that corresponds to him. Guaranteeing the adequate use of hospital resources and reducing the waiting list for a first in-person visit in rheumatology in primary care is one of the most important goals fulfilled, given that it is directly related to maintaining the accessibility and equity of the public health system.

\section{REFERENCES:}

[1] Kataria, S., \& Ravindran, V. (2018). Digital health: a new dimension in rheumatology patient care. Rheumatology International. https://doi.org/ 10.1007/s00296-018-4037-

Tabla 1: Resolution of the first virtual visits to 6 months of REVIR project

\begin{tabular}{lcccc}
\hline CAP & Total & First CAP & First Hospital & Virtual resolution \\
\hline Ramón Turró & $193(26,4 \%)$ & $94(49 \%)$ & $22(11,3 \%)$ & $77(40 \%)$ \\
Villa Olímpica & $96(13,2 \%)$ & $36(37,5 \%)$ & $13(13,5 \%)$ & $47(49 \%)$ \\
Sant Martí (1 y 2) & $437(60,2)$ & $216(49,5 \%)$ & $53(12,1 \%)$ & $168(38,4 \%)$ \\
Total & $726(100 \%)$ & $346(47,6 \%)$ & $88(12,1 \%)$ & $292(40,2 \%)$ \\
\hline
\end{tabular}

Disclosure of Interests: Fabiola Ojeda: None declared, Manel Ciria: None declared, Carolina Perez-Garcia: None declared, Eric Sitjas: None declared, Elena Martinez: None declared, Daniel Martinez-Laguna Speakers bureau: Eli Lilly, Amgen, Ferrer, Rubió and Novartis., Montserrat Pimienta: None declared, Jordi Monfort: None declared DOI: 10.1136/annrheumdis-2019-eular.4250

\section{THU0646 HIGH LEVELS OF DAMAGE IN INFLAMMATORY RHEUMATIC DISEASES: A CLUE TO LOW RATES OF REMISSION AND LOW DISEASE ACTIVITY}

Isabel Castrejon, Sarah Everakes, Joel Block, Sonali Khandelwal, Ailda Nika, Padmanabhan Raghu, Theodore Pincus. Rush University Medical Center, Rheumatology, Chicago, United States of America

Background: Despite introduction of powerful biologic medications over the last 2 decades, rates of remission and low disease activity rates in RA remain less than $50 \%$. One possible basis is that measures and indices such as disease activity score 28 (DAS28) and clinical disease activity index (CDAl), while sensitive primarily to disease activity in clinical trial patients selected for high inflammatory activity, may also reflect clinically important joint damage and patient distress in unselected patients in routine care. Similar considerations may pertain to systemic lupus erythematosus disease activity index (SLEDAl), Bath ankylosing spondylitis disease activity index (BASDAI), and other measures and indices initially designed to assess disease activity. Levels of organ damage and patient distress, as well as inflammation, may be quantitated according to 3 physician (0-10) visual analog scales (VAS), in addition to physician global assessment VAS (DOCGL), scored in fewer than 10 seconds in routine care.

Objectives: To test a hypothesis that damage and distress may be prominent in patients with inflammatory conditions, according to mean VAS for inflammation or reversible findings (DOCINF), damage or irreversible findings (DOCDAM), and distress (DOCSTR), e.g., fibromyalgia.

Methods: All patients at one site complete a multidimensional health assessment questionnaire (MDHAQ), which includes patient global VAS (PATGL), at each visit in routine care. Physicians complete four 0-10 (none-highest) VAS for DOCGL, DOCINF, DOCDAM, and DOCSTR, and a query to estimate the proportion of clinical decisions (total=100\%) attributed to each of the 3 findings. Patients were classified into various diagnostic groups, in which scores were analyzed according to mean and standard deviation.

Results: Analyses included 563 patients (Table). Mean levels of DOCGL ranged from 3.2 to 5.2, and PATGL from 3.6 to 6.5 , which might be interpreted to indicate high disease activity. Highest mean DOCINF scores were seen in patients with RA, SLE, vasculitis, polymyalgia rheumatica (PMR), spondyloarthropathy (SpA), and gout (2.2-2.8), while highest mean DOCDAM was seen in OA (4.9) and DOCSTR in FM (6.2) (Table). How ever, in RA, mean DOCDAM was 3.7 vs 2.4 for DOCINF. DOCDAM also was almost as high or higher than DOCINF in SLE, SpA, vasculitis, and gout. Mean estimates of distress were also $>1.5$ in patients with all inflammatory diagnoses.

\begin{tabular}{|c|c|c|c|c|c|c|}
\hline \multirow[t]{2}{*}{$\begin{array}{l}\text { Diagnosis (per ICD } \\
\text { code) }\end{array}$} & \multirow[t]{2}{*}{$\begin{array}{l}\mathbf{N} \\
(\%)\end{array}$} & \multicolumn{2}{|c|}{$\begin{array}{c}\text { Global Estimates } \\
(0-10)\end{array}$} & \multicolumn{3}{|c|}{ Physician subscales $(0-10)$} \\
\hline & & $\overline{\text { PATGL }}$ & DOCGL & DOCINF & DOCDAM & DOCSTR \\
\hline $\mathrm{RA}$ & 64 & $\begin{array}{l}4.9 \\
(2.9)\end{array}$ & $4.5(2.4)$ & $2.4(2.2)$ & $3.7(2.8)$ & $1.9(2.7)$ \\
\hline SLE & 47 & $\begin{array}{l}4.1 \\
(3.1)\end{array}$ & $3.7(2.8)$ & $2.2(2.4)$ & $1.5(1.7)$ & $1.8(2.5)$ \\
\hline SpA and psoriatic arthritis & 34 & $\begin{array}{l}4.1 \\
(2.9)\end{array}$ & $3.9(2.4)$ & $2.4(2.5)$ & 2.3) & $1.9(2.8)$ \\
\hline Vasculitis and PMR & 23 & $\begin{array}{l}3.6 \\
(2.6)\end{array}$ & $3.4(3.0)$ & $2.6(1.8)$ & $2.3(2.2)$ & $1.7(2.6)$ \\
\hline Gout & 21 & $\begin{array}{l}4.4 \\
(2.9)\end{array}$ & 4.2 & $2.8(2.5)$ & 2.8) & $1.5(1.9)$ \\
\hline Osteoarthritis & 135 & $\begin{array}{l}5.8 \\
(2.9)\end{array}$ & $4.9(2.2)$ & $1.2(1.9)$ & $4.9(2.5)$ & $2.2(2.7)$ \\
\hline Fibromyalgia & 84 & $\begin{array}{l}6.5 \\
(2.6)\end{array}$ & $5.2(2.2)$ & $0.9(1.2)$ & $1.7(2.0)$ & $6.2(2.6)$ \\
\hline Other diagnosis & 155 & $\begin{array}{l}4.0 \\
(3.0)\end{array}$ & $3.2(2.2)$ & $1.6(2.0)$ & $2.4(2.3)$ & $1.6(2.1)$ \\
\hline TOTAL & 563 & $\begin{array}{c}4.8 \\
(3.0)\end{array}$ & $3.9(2.4)$ & $1.6(2.0)$ & $2.9(2.5)$ & $2.4(2.9)$ \\
\hline
\end{tabular}

Conclusion: Rheumatologists estimated high levels of damage in patients with RA and other inflammatory rheumatic diseases, similar or higher than inflammation, as well as recognizable distress which may elevate measures such as tender joint count and PATGL. These findings may explain in part low rates of remission and low disease activity noted in RA and other inflammatory diseases, as index scores used to document improvement are not affected by anti-inflammatory therapy. Most rheumatology clinical quantitative measurement is directed to inflammatory activity. However, an estimate of damage and distress may clarify why many patients may appear to have suboptimal control of inflammatory activity despite aggressive treatment, including treat to target in RA.

Disclosure of Interests: None declared

DOI: 10.1136/annrheumdis-2019-eular.3331 